VOL. 60 (1999) [335-336]

\title{
On classes associated with radical and semisimple classes
}

\author{
RoBert McDougaLL
}

This thesis details an investigation into some aspects of the nature of radical and semisimple classes of associative rings. It deals particularly with the search and construction of pairs of radical classes which have no common rings, the classes of rings that can generate radical and semisimple classes and the relationship between constructions in radical theory.

Beginning with an investigation into pseudocomplements and complements in the lattice of all radical classes, the notion of a pseudocomplemented pair is introduced and examples of pseudocomplements in the lattice of all radical classes using the characterisation of the pseudocomplement for hereditary radical classes and an upper and lower bound for the pseudocomplement for any radical class $\mathcal{R}$ are given. An example of a pseudocomplement involving the strongly $\mathcal{R}$-semisimple rings and a condition for the termination of successive applications of the upper radical operator is included.

The discussion is then extended and complemented pairs of radical classes are investigated. A characterisation of complemented hereditary radical classes in the lattice of all radical classes in terms of their semisimple closure is given. Realising that complemented radical classes are complemented by their pseudocomplement uncovers a class of nonhereditary complements in the lattice. Some breadth is added to the recently confirmed results that the only complemented hereditary radical classes in the lattice of all radical classes are those complemented in its sublattice, the lattice of all hereditary radical classes.

The discussion then evolves into a systematic examination of two constructions which under appropriate well known conditions generate classes of rings related to the pseudocomplements and complements mentioned earlier. The first is a straightforward construction generating a semisimple class requiring no special conditions on the generating class. It is equivalent to the semisimple closure under certain conditions but is quite a different semisimple class under others. The semisimple class generated is related to the pseudocomplement of radical class in the lattice of all radicals and a

Received 27th april, 1999

Thesis submitted to the Central Queensland University, October 1998. Degree approved, April 1999. Supervisor: Dr N.R. McConnell.

Copyright Clearance Centre, Inc. Serial-fee code: 0004-9727/99 \$A2.00+0.00. 
characterisation of regular radical classes that are complemented in this lattice is given. Although we focus on radical classes of associative rings, we expect this construction to have wider appeal and application in a variety of mathematical settings. The second is a one-step construction generating the largest hereditary radical class having zero intersection with the class that determines it. An interesting result that uses the construction to generate a radical class that can be described in a similar way to the usual lower radical class construction but determined by a class which is never homomorphically closed motivates a discussion on to the nature of the constructions used in radical theory and their interrelationship.

That the lower radical class construction on a homomorphically closed class of associative rings will generate a radical class for any class of associative rings is then demonstrated and a discussion of some of the consequences of this generalisation follows. A new description of the upper radical class using this construction on an appropriate generating class is included.

Finally, the lower and upper radical class constructions and the duality existing between the concepts of homomorphic image and accessible subring allow a tour of many well known classes of rings to be made. The generality of the work gives some new information about the nature and consequences of the various constructions in radical theory.

School of Mathematical and Decision Sciences

Faculty of Informatics and Communication

Central Queensland University

Bruce Highway

North Rockhampton, Qld 4702

Australia 